Pesq. Vet. Bras. 30(4):328-334, abril 2010

\title{
Segurança e eficácia da técnica de biopsia pulmonar transtorácica percutânea para avaliação histopatológica do parênquima pulmonar de ovinos clinicamente sadios ${ }^{1}$
}

\author{
Andreza Amaral da Silva ${ }^{2 \star}$, Danilo Otávio Laurenti Ferreira² ${ }^{2}$ Camila Dias Porto ${ }^{3}$, \\ Bianca Paola Santarosa ${ }^{4}$, Júlio Lopes Sequeira ${ }^{5}$ e Roberto Calderon Gonçalves ${ }^{5}$
}

\begin{abstract}
Silva A.A., Ferreira D.O.L., Porto C.D., Santarosa, B.P., Sequeira J.L. \& Gonçalves R.C. 2010. [Safety and effectiveness of the transthoracic percutaneous lung biopsy technique for histopathological evaluation of the lung parenchyma in healthy sheep.] Segurança e eficácia da técnica de biópsia transtorácica percutânea para avaliação histopatológica do parênquima pulmonar de ovinos clinicamente sadios. Pesquisa Veterinária Brasileira 30(4):328-334. Departamento de Clínica Veterinária, Faculdade de Medicina Veterinária e Zootecnia, Universidade Estadual Paulista, Distrito de Rubião Júnior s/n, Botucatu, SP 18618-000, Brazil. E-mail: andrezamedvet@yahoo.com.br

The results and complications arising from use of the percutaneous transthoracic lung biopsy technique in 20 clinically healthy sheep were analyzed. The animals were subjected to clinical examination followed by lung biopsy with a semi-automatic needle in the $7^{\text {th }}$ right intercostal space, $5 \mathrm{~cm}$ above the olecranon. The number of attempts to implement the procedure and size of the fragments were evaluated. The samples were evaluated histologically. After the slaughter, a postmortem examination assessed the technical complications and possible injuries. Among all animals which underwent biopsy only two did not tolerate the technique. Lung fragments measuring $4-7 \mathrm{~mm}$ were obtained by an average of 1.8 attempts per fragment. The main changes related to the procedure were cough, labored breathing, increased heart and breathing rate, and increased bronco-bronchiolar sound. The postmortem examination revealed bleeding of the intercostal muscles and visceral pleura of various extend in all lambs, and a wound up to $14 \mathrm{~mm}$ length in the lung of two animals that did not tolerate the procedure. From 20 attempts to implement the technique, 18 were successful, while in two of them the liver was mistakenly punctured. Samples of lung tissue were representative. It was possible to visualize intact structures, including bronchioles and alveoli. It could be concluded that lung biopsy is a safe and effective procedure to obtain lung samples for histological diagnosis.
\end{abstract}

INDEX TERMS: Lung biopsy, auxiliary tests, lung, sheep.

${ }^{1}$ Recebido em 2 de março de 2009.

Aceito para publicação em 28 de novembro de 2009.

Dissertação de Mestrado do primeiro autor. Auxílio financeiro (Proc. 07/55341-6) e bolsa de mestrado (Proc. 07/51713-6) da Fundação de Amparo à Pesquisa do Estado de São Paulo (FAPESP).

${ }^{2}$ Mestrando(a) do Programa de Pós-Graduação em Medicina Veterinária, Departamento de Clínica Veterinária, Faculdade de Medicina Veterinária e Zootecnia (FMVZ), Unesp, Campus de Botucatu, Distrito de Rubião Júnior s/n, Botucatu, SP 18618-000, Brasil. "Autor para correspondência: andrezamedvet@yahoo.com.br

3 Doutoranda do Programa de Pós-Graduação em Medicina Veterinária, FMVZ, Unesp, Campus de Botucatu, SP.

${ }^{4}$ Graduanda em Medicina Veterinária da FMVZ, Unesp, Campus de Botucatu, SP.

${ }^{5}$ Departamento de Clínica Veterinária da FMVZ, Unesp, Campus de Botucatu, SP.
RESUMO.- Foram analisados os resultados e complicações decorrentes do emprego da técnica de biopsia pulmonar transtorácica percutânea em 20 ovinos clinicamente sadios. Os animais foram submetidos ao exame clínico seguido da biopsia com agulha semi-automática no $7^{\circ}$ espaço intercostal direito, $5 \mathrm{~cm}$ acima do olécrano. Foram analisados o número de tentativas para a execução do procedimento e o tamanho dos fragmentos. As amostras obtidas foram avaliadas histologicamente. Posteriormente ao abate, foi realizado o exame pos mortem para avaliação de complicações da técnica e das eventuais lesões provocadas. Entre todos os animais submetidos à biopsia apenas dois demonstraram resistência a técnica, sendo obtidos fragmentos pulmonares de 4-7mm de comprimen- 
to, com média de $1,8 \pm 1$ tentativas por fragmento. As principais alterações relacionadas à técnica foram tosse, dispnéia inspiratória, elevação das freqüências cardíaca e respiratória e aumento do ruído broncobronquiolar. Ao exame post mortem observou-se hemorragia dos músculos intercostais e pleura visceral em todos os animais. Das 20 tentativas de execução da técnica, 18 obtiveram sucesso, enquanto que em duas o fígado foi equivocadamente puncionado. As amostras de tecido pulmonar foram consideradas representativas, pois foi possível a visualização de estruturas íntegras, incluindo bronquíolos e alvéolos. Podemos concluir que a biopsia pulmonar é segura e eficaz para obtenção de amostras pulmonares com fins de diagnóstico histológico.

TERMOS DE INDEXAÇÃO: Biopsia pulmonar, exame complementar, pulmão, ovinos.

\section{INTRODUÇÃO}

As doenças que afetam o trato respiratório inferior são consideradas a principal causa de perda na ovinocultura moderna (Dohoo et al. 1985, Martin 1996, Cutlip et al. 1998), sendo responsáveis por $10-40 \%$ das mortes em animais adultos (Vieira et al. 1993) e por $17 \%$ das mortes durante 0 período perinatal (Rook et al. 1990).

Apesar de ocorrerem com freqüência em rebanhos ovinos, muitas vezes as causas dessas enfermidades pulmonares não podem ser determinadas apenas com a realização de um exame clínico mais acurado, sendo necessários outros recursos diagnósticos para definir sua etiologia. Além disso, a necessidade de diagnóstico específico das doenças pulmonares é uma imposição dos recursos terapêuticos atuais.

A biópsia pulmonar é um procedimento diagnóstico invasivo, geralmente utilizado quando outros procedimentos menos invasivos como radiografia, lavado traqueobrônquico e/ou broncoalveolar, endoscopia e ultrassonografia já foram realizados sem uma definição diagnóstica, ou quando há necessidade de um diagnóstico rápido e preciso (Porto 1991). A avaliação histológica de fragmentos pulmonares obtidos através da biopsia é útil para caracterização de danos superficiais e subpleurais e para diferenciar lesões inflamatórias, alérgicas, enfisematosas, edematosas e neoplásicas (Braun et al. 1999). Além disso, as amostras de pulmão podem ser submetidas à cultura de fungos $\mathrm{e}$ bactérias, bem como testes de sensibilidade a antibióticos, dependendo da suspeita diagnóstica (Savage et al. 1998). Entretanto, poucos estudos relatando a segurança e eficácia da biópsia pulmonar em ovinos já foram publicados.

O primeiro relato científico de biopsia pulmonar em ovinos foi de Pusterla et al. (1995), descrevendo a utilização da técnica para a obtenção de fragmentos pulmonares de animais com adenomatose pulmonar. Em seguida houve dois relatos de biopsia pulmonar aspirativa guiada por ultrasom (Braun et al. 1995, Scott \& Gessert 1998) e outro de biopsia pulmonar lancetante guiada por ultra-som (Braun et al. 2000).
O objetivo deste estudo é avaliar a segurança da técnica de biopsia pulmonar percutânea transtorácica às cegas e sua eficácia na obtenção de amostras adequadas que permita a análise histopatológica do fragmento pulmonar.

\section{MATERIAL E MÉTODOS}

Foram utilizados 20 ovinos clinicamente sadios (13 da raça Santa Inês e 7 mestiços Santa Inês), 9 machos e 11 fêmeas, com idade de 150-190 dias (média de 175 dias), pesando 23$44 \mathrm{~kg}$ (média $32 \mathrm{~kg}$ ) e destinados ao abate. Todos os animais foram submetidos ao exame clínico imediatamente antes e após a biopsia pulmonar, utilizando-se protocolo adotado por Viana (2003), e critério clínico de Gonçalves (2004). Os resultados foram anotados em fichas individuais.

O procedimento foi realizado com os animais em estação e contidos manualmente com a ajuda de um assistente. $O$ ponto de punção para a biopsia pulmonar foi demarcado no sétimo espaço intercostal do antímero lateral direito, $5 \mathrm{~cm}$ acima do olécrano. Esse ponto corresponde à posição topográfica do lobo caudal do pulmão direito. A seguir foi feita tricotomia e antissepsia da pele com solução de iodo povidine a $1 \%$, seguido de anestesia local da pele do espaço intercostal, tecido subcutâneo, músculos intercostais e pleura parietal, utilizandose $5 \mathrm{ml}$ de solução de lidocaína a $2 \%$. Alguns minutos depois foi feita, com bisturi, incisão de aproximadamente $1 \mathrm{~cm}$ na pele e tecido subcutâneo, entre os espaços intercostais. Uma agulha de biopsia do tipo semi-automática (BD $16 \mathrm{G} \times 15 \mathrm{~cm}$; Euromed Cateteres $^{\text {}}$ ) foi inserida, imediatamente cranial à costela, em ângulo vertical à superfície corporal, evitando-se os vasos e nervos intercostais.

Amostras do tecido pulmonar foram colhidas de acordo com Finn-Bodner \& Hathcock (1993) e Braun et al. (1999). Se nenhum fragmento do parênquima fosse obtido após a punção, o procedimento seria repetido até que se conseguisse uma amostra satisfatória do tecido pulmonar. Foi analisado o número de tentativas para a execução do procedimento. Imediatamente após a punção, os fragmentos pulmonares obtidos foram acondicionados em recipientes contendo formalina a $10 \%$ e, posteriormente, desidratados em álcool, embebidos em parafina, seccionados e corados com hematoxilina-eosina.

Nenhuma medicação foi administrada aos animais e nenhuma atenção especial foi dada ao sítio da biopsia, uma vez que todos os animais foram submetidos ao abate logo após o procedimento.

Os animais foram analisados por inspeção no exame post mortem para avaliação de complicações decorrentes da técnica. Hemorragias na pleura visceral atribuídas à biopsia foram avaliadas segundo o escore de gravidade da lesão descrito por Silva (2005), adaptado de Braun et al. (1999) (escore 1 = lesão pleural com área até $4 \mathrm{~mm}^{2}$; escore 2 = lesão pleural com área entre 4,1 e $25 \mathrm{~mm}^{2}$; escore 3 = lesão pleural com área superior a $\left.25,1 \mathrm{~mm}^{2}\right)$. As amostras de tecido pulmonar obtidas também foram submetidas ao exame macroscópico, avaliando-se o comprimento de cada fragmento tecidual.

A avaliação histológica dos fragmentos de biopsia foi realizada utilizando-se microscópio binocular convencional. A qualidade dos cortes histológicos obtidos foi avaliada segundo escore adaptado de Hopper et al. (1990) para: 1) adequação tecidual para o diagnóstico histopatológico (escore 0 = tecido insuficiente para análise histopatológica; escore $1=$ tecido 
insuficiente para uma avaliação histopatológica adequada, mas é possível um diagnóstico altamente suspeito; escore 2 = tecido suficiente para a avaliação histopatológica); 2) Preservação Tecidual (escore 0 = pobre; escore 1 = intermediária; escore 2 = boa); 3) Presença de Artefatos (escore 0 = intensa; escore 1 = intermediária; escore 2 = sem presença de artefatos).

Os dados coletados foram expostos por meio de estatística descritiva, através de média, desvio padrão e percentual.

\section{RESULTADOS}

Pela técnica anteriormente descrita, fragmentos teciduais foram obtidos em todos os animais submetidos ao procedimento (20/20; 100\%), sendo necessária, em média 1,8 11 tentativas. Das 20 biopsias realizadas, amostras de tecido foram obtidas após apenas uma tentativa em nove ocasiões (9/20; 45\%), após duas tentativas em sete (7/ $20 ; 35 \%)$, após três tentativas em dois momentos (2/20; $10 \%)$ e, após 4 tentativas também em dois (2/20; $10 \%)$ momentos. A agulha escolhida foi de fácil manipulação e a técnica foi executada sem grandes dificuldades.

Treze animais (13/20; 65\%) toleraram bem a técnica de biopsia pulmonar transtorácica percutânea, entretanto, alguns animais demonstraram certa resistência durante o procedimento, vindo até a dificultar a coleta. Cinco (5/ $20 ; 25 \%$ ) animais demonstraram suave esforço (berros, feições de medo e elevação das patas), enquanto que dois $(2 / 20 ; 10 \%)$ animais apresentaram forte resistência (saltos e tentativas de coices) à realização da técnica.

Ao exame clínico, atitude geral e postura permaneceram inalteradas e a temperatura retal não se elevou, permanecendo dentro dos valores de referência para a espécie. Entretanto, as freqüências cardíaca e respiratória aumentaram em 14 (70\%; Média (MED) = 122bpm) e 12 $(60 \%$; MED $=52 \mathrm{mrp})$ animais, respectivamente. Apesar disso, os parâmetros observados voltaram à normalidade em aproximadamente 15 minutos após a biopsia.

Com relação a alterações no aparelho respiratório, foi observada tosse espontânea em seis $(6 / 20 ; 30 \%)$ animais. Anormalidades no padrão respiratório ocorreram em 3 (3/ $20 ; 15 \%)$ ovinos, sendo que dois deles apresentaram dispnéia inspiratória e um animal apresentou expiração em dois tempos, o que geralmente é característico de dor. À auscultação pulmonar, foi observado aumento do ruído broncobronquiolar no sítio da biopsia, em três (3/20; 15\%) animais. Os dois ovinos que demonstraram forte resistência à execução do procedimento apresentaram, à auscultação pulmonar no local da biopsia, um som característico de vazamento de ar, exatamente sobre o local da punção, embora sem haver saída de ar para o exterior.

Um animal entrou em colapso imediatamente após a realização da biopsia, vindo a decúbito. Este animal apresentou tosse, taquicardia, taquipnéia e intensa dispnéia inspiratória, adotando postura ortopneica.

A auscultação pulmonar deste animal revelou aumento do ruído broncobronquiolar por toda área dos pulmões. Apesar disso, o animal levantou em alguns minutos e não apresentou mais nenhuma anormalidade. Nenhum dos animais apresentou hemoptise, epistaxe ou mesmo sinais clínicos compatíveis com hemotórax ou pneumotórax, complicações comumente associadas à biopsia pulmonar.

Os animais foram abatidos, em média, três horas após a realização da biopsia pulmonar.

No exame post mortem observou-se que todas as punções estavam localizadas no lobo caudal. Das 20 biopsias realizadas, as alterações encontradas na pleura parietal e músculos intercostais eram evidenciados como um ponto em 12 carcaças (12/20; $60 \%$ ); em outras quatro (4/20; $20 \%$ ) as lesões tinham até $4 \mathrm{~mm}$ de comprimento; foram evidenciadas como grandes hematomas de até $8 \times 10 \mathrm{~mm}$ em quatro carcaças $(4 / 20 ; 20 \%)$. Todos os animais apresentaram hemorragia de pleura, cuja área variou de $4 \mathrm{~mm}^{2}$ a $40 \mathrm{~mm}^{2}$. Dos 20 animais biopsiados, avaliados quanto ao escore de severidade da hemorragia pleural proposto por Silva (2005), adaptado de Braun et al. (1999), oito ovinos $(8 / 20 ; 40 \%)$ apresentaram escore 1 , seis $(6 / 20$; $30 \%)$ apresentaram escore 2 e outros seis animais (6/20; $30 \%$ ) apresentaram escore 3 . Ao exame macroscópico dos pulmões foram observados petéquias e enfisema ao redor da ferida da biopsia, presentes em quatro $(4 / 20 ; 20 \%)$ e cinco $(5 / 20 ; 25 \%)$ ovinos, respectivamente. Dois animais, mais precisamente os que demonstraram forte resistência durante a execução da biopsia, apresentaram uma ferida linear de aproximadamente $12 \mathrm{~mm}$ e $14 \mathrm{~mm}$, respectivamente, na face costal do pulmão direito na região basal do lobo caudal. No pulmão de dois ovinos foi observado sinais de que a agulha de biopsia havia transpassado o lobo caudal.

Os fragmentos teciduais obtidos através da biopsia variaram de 4 a $17 \mathrm{~mm}$ de comprimento (MED $=10 \mathrm{~mm})$. Das 20 amostras obtidas 18 (18/20; 90\%) apresentaram tecido pulmonar íntegro e em quantidade representativa e duas (2/20; $10 \%)$ eram compostas somente de tecido hepático, sendo consideradas impróprias para a avaliação pulmonar. Em cinco casos $(5 / 18 ; 28 \%)$, além de pulmão, também foram observados pequenos fragmentos de pele, músculo esquelético, fígado ou tecido conjuntivo de origem indeterminada. Contudo, a presença desses fragmentos não comprometeu a avaliação histológica pulmonar, sendo considerados artefatos iatrogênicos. Todas as amostras de pulmão $(18 / 18 ; 100 \%)$ estavam adequadas para análise histopatológica por apresentarem arquitetura pulmonar preservada. Quatorze amostras (14/18; 78\%) foram consideradas ideais para avaliação histológica, pois foi possível observar bronquíolos primários, terminais e respiratórios, ductos e sacos alveolares bem como alvéolos isolados. Em 4 amostras (4/18; 22\%) não foi possível encontrar bronquíolos, estando ausentes inclusive os bronquíolos terminais. Nestas lâminas estavam presentes apenas ductos e sacos alveolares e alvéolos isolados e por isso foram classificadas apenas como boas para uma avaliação histológica. Duas amostras $(2 / 18 ; 11 \%)$ apresentaram infiltrado inflamatório misto discreto, composto por mononucleares e neutrófilos. Dois fragmentos $(2 / 18 ; 11 \%)$ teciduais apresentaram focos hemorrágicos isolados. 
Um dos fragmentos pulmonares $(1 / 18 ; 5 \%)$ apresentou aumento da celularidade do interstício e conseqüente espessamento, com infiltrado inflamatório misto moderado, composto por mononucleares e eosinófilos, além de focos hemorrágicos. A luz bronquiolar mostrou-se diminuída, com presença de debris celulares. A pleura, observada em uma das extremidades da biópsia, exibiu espessamento. Esses achados são compatíveis com 0 diagnóstico de pneumonia intersticial crônica.

Todas as amostras de pulmão obtidas (18/18; 100\%) mostraram áreas atelectásicas nas extremidades e duas amostras $(2 / 18 ; 11 \%)$ apresentaram também esmagamento tecidual com comprometimento de parte da área amostrada, todavia nenhuma dessas alterações comprometeu a avaliação morfológica.

A qualidade dos cortes histológicos pulmonares obtidos foram avaliados segundo o escore adaptado de Hopper et al. (1993), que considera os graus zero, um e dois qualitativamente. Levando em conta a adequação tecidual, todas as amostras (18/18; 100\%) apresentaram escore 2; com relação a preservação tecidual, duas amostras $(2 / 18 ; 11 \%)$ apresentaram escore 1 e, 16 amostras (16/8; 89\%) obtiveram escore 2; analisando a presença de artefatos, em todas as lâminas confeccionadas (18/ 18; 100\%) verificou-se escore 1.

\section{DISCUSSÃO}

A biopsia pulmonar percutânea realizada com agulhas lancetantes demorou para ganhar aceitação, mas o avanço nas técnicas de biopsia, melhorias nas agulhas e a possibilidade de lidar melhor com as complicações decorrentes da técnica contribuíram para o aumento da popularidade desta útil ferramenta de auxílio diagnóstico. Neste estudo, as biopsias pulmonares foram conduzidas às cegas de forma que houvesse máxima probabilidade de se conseguir amostras de tecido viáveis para um diagnóstico histológico quando fosse aplicada em ovinos com doença pulmonar difusa. Se o objetivo do procedimento for obter fragmentos teciduais de lesões localizadas, a biopsia pulmonar deve ser preferencialmente, guiada por ultrasom ou radiografias, como já relatado por outros autores (Wood et al. 1998, Braun et al. 2000).

Foram necessárias em média 1,8 tentativas até a obtenção de um fragmento de tecido viável para análise histológica. Outros autores relatam resultados semelhantes, com média de 1,2 para Braun et al. (2000) que também utilizaram uma agulha semi-automática em ovinos, e de 1,3 para Hamarati (1995) que utilizou um dispositivo automático em humanos. Porém, nestes dois casos as biopsias não foram realizadas às cegas. A execução da biopsia pulmonar às cegas aumenta o risco de complicações como punção equivocada de outros órgãos, lesões de grandes vasos e morte em conseqüência de hemorragias. No presente estudo foram necessárias poucas perfurações para a obtenção de amostras de tecido viáveis para um exame histológico e não foram observadas complicações graves em decorrência da técnica, indicando que, nestas condições, o $7^{\circ}$ espaço intercostal é um bom acesso para a cavidade torácica em ovinos, especialmente quando se pretende obter amostras do lobo caudal.

Segundo Moore (2005) e Braun et al. (1999) as reações de desconforto e dor ocorrem durante a perfuração da pleura parietal e não durante a coleta do tecido pulmonar. Por isso, a perfuração da pleura deve ser bem rápida para eliminar a possibilidade de que qualquer movimento repentino desvie a trajetória da agulha ou então que sua ponta cortante lese a pleura antes da colheita de tecido pulmonar. Dois animais deste estudo demonstraram forte resistência à técnica, com reações semelhantes às relatadas em bovinos por Braun et al. (1999), principalmente no momento da perfuração da pleura. Ao exame pos mortem pôde-se observar perfurações lineares de até $14 \mathrm{~mm}$ nos pulmões desses ovinos. Embora estivessem anestesiados, é possível que o volume da droga injetado tenha sido insuficiente para uma adequada anestesia da pleura parietal, e que o aumento na quantidade de anestésico administrado possa reduzir a dor desta perfuração, como já foi relatado para eqüinos (Raphel \& Gunson 1981) e bovinos (Braun et al.1999). Outra opção para evitar reações bruscas dos animais que possam atrapalhar o bom andamento da técnica seria o uso de sedativos. Contudo, antes da indicação deste tipo de fármaco é necessária uma avaliação minuciosa da função pulmonar dos animais, geralmente comprometida em casos de doença respiratória grave.

As alterações observadas ao exame clínico conduzido após a biopsia pulmonar estão de acordo com as descrições prévias da literatura (Raphel \& Gunson 1981, Savage et al. 1998, Braun et al. 1999, 2000, Silva 2005). O aumento das freqüências cardíaca e respiratória, observadas em alguns animais, deve ter ocorrido em conseqüência ao estresse da contenção e da realização do procedimento, visto que esses parâmetros voltaram à normalidade dentro de poucos minutos após a realização da biopsia.

Com relação ao exame do aparelho respiratório, a elevação do ruído broncobronquiolar provavelmente ocorreu em decorrência do esforço físico de alguns animais ao resistirem à execução da biopsia. A dispnéia inspiratória, observada em dois animais, poderia ser resultado da agressão da agulha ao tecido pulmonar, o que pode determinar um sangramento no parênquima pulmonar (Radostits et al. 2002). Entretanto, nenhum dos animais que apresentaram dispnéia inspiratória após o procedimento apresentou focos hemorrágicos na avaliação histológica da amostra obtida. Por outro lado, uma hemorragia focal, como foi observada, não seria suficiente para causar alteração no padrão respiratório. Pode-se deduzir que a biopsia não determina grande modificação na estrutura pulmonar, mas que 0 ato de contenção associado com a dor conseqüente à perfuração pleural poderia levar à dispnéia inspiratória observada nesses dois animais. Se esses dois ovinos apresentassem problema decorrente da lesão provocada pela biopsia provavelmente 
haveria manutenção da dificuldade respiratória, o que não foi observado já que todos os animais voltaram ao seu padrão respiratório normal em aproximadamente quinze minutos após o procedimento, indicando a segurança da técnica.

A auscultação pulmonar do sítio da biopsia revelou, em dois animais, um ruído semelhante a vazamento de ar (sopro). Este tipo de ruído provavelmente ocorreu pela perfuração da pleura visceral e do tecido pulmonar subjacente com saída de ar para a cavidade pleural, já que esses animais foram os que apresentaram maior resistência ao procedimento. A entrada de ar na cavidade pleural pode levar a um pneumotórax, se a capacidade de absorção pleural for ultrapassada (Porto 1991). Os animais deste estudo não apresentaram qualquer sinal clínico compatível com pneumotórax após a realização do procedimento. Entretanto, pneumotórax de pequenas proporções e assintomático já foi relatado em bovinos e humanos submetidos à biopsia pulmonar (Braun et al. 1999, Geraghty et al., 2003). Como não foi feito diagnóstico por acompanhamento radiográfico (Braun et al. 1999, Venner et al. 2006) ou por auscultação seriada algumas horas após a biopsia, já que os animais precisavam ser abatidos, o pneumotórax, caso tenha ocorrido, não pode ser constatado.

O motivo para o colapso momentâneo ocorrido logo após a biopsia pulmonar em um dos animais não foi determinado, embora reações como essa já tenha sido relatada por outros autores em bovinos, também sem causa aparente (Braun et al. 1999). Contrariando observações de outros autores que apontam a hemoptise e a epistaxe como complicações normalmente associadas à biopsia pulmonar (Raphel \& Gunson 1981, Savage et al. 1998; DeBerry et al. 2002, Silva 2005, Venner et al. 2006), não foram observados nesta pesquisa sinais de sangramento em nenhum dos animais após o procedimento. Entretanto, Venner et al. (2006) relatam que indícios de hemorragia são geralmente evidenciados após endoscopia, mesmo em animais que não apresentaram sinais aparentes de sangramento. Neste estudo, o exame post mortem não revelou estrias de sangue ou coágulos no trato respiratório de nenhum dos animais, o que elimina as chances de que hemorragias de grande porte tenham realmente ocorrido. Contudo, foram observadas petéquias próximas ao local da punção nos pulmões de dois ovinos, indicando que pequenas hemorragias no parênquima pulmonar sem importância clínica ocorreram em conseqüência da biopsia, como já observado por outros autores (Roy \& Lavoie 2003).

As hemorragias de pleura visceral ocorreram em todos os ovinos, sendo semelhantes às previamente relatadas em outros estudos (Braun et al. 1999, 2000, Silva 2005, Venner et al. 2006). Essas hemorragias foram agrupadas de acordo com o escore atribuído a área total das lesões. O escore de lesão adotado para a biopsia neste estudo foi inferior aos resultados descritos por Silva (2005) e superiores aos de Braun et al. (1999). Silva (2005) tra- balhou com uma agulha de biopsia de grosso calibre, o que provavelmente ocasionou maior dano tecidual que a agulha de médio calibre utilizada no presente trabalho. Braun et al. (1999), por sua vez, determinou o escore da lesão 10 dias após a realização da biopsia, havendo, dessa forma, tempo hábil para reparação tecidual. As feridas lineares de até $14 \mathrm{~mm}$ observadas no parênquima pulmonar de dois ovinos ocorreram provavelmente em conseqüência da agitação demonstrada por esses animais no momento da biopsia.

Os tamanhos dos fragmentos teciduais obtidos neste estudo estão de acordo com as descrições prévias da literatura (Braun et al. 1999, 2000, Silva 2005). A técnica empregada permitiu a avaliação da arquitetura tecidual pulmonar. Uma biopsia é considerada representativa quando são visualizadas unidades funcionais do tecido amostrado (Naylor et al. 1992). Neste estudo foi possível observar em aproximadamente $80 \%$ das amostras obtidas, bronquíolos primários, terminais e respiratórios, ductos e sacos alveolares, bem como alvéolos isolados. Resultados semelhantes também foram observados em eqüinos (Raphel \& Gunson 1981, Venner 2006), bovinos (Dungworth \& Hoare 1970, Braun et al. 1999) e ovinos (Braun et al. 2000). Não foi possível verificar a presença de brônquios nas amostras obtidas devido a provável ausência dessas estruturas no local onde foi realizada grande parte das punções, a porção basal do lobo caudal.

Duas amostras apresentaram perfil histológico compatível com processo inflamatório muito discreto, composto por mononucleares e neutrófilos. Uma possível explicação para esse achado é que, se estes animais realmente apresentavam doença respiratória, o processo ainda estava muito incipiente para ser detectado ao exame clínico. Como os animais desta espécie, devido as condições de manejo a que são submetidos, vivem constantemente expostos a fatores de risco, é aceitável que achados como este ocorram quando se realiza biopsia pulmonar. Os focos hemorrágicos encontrados em duas lâminas foram conseqüentes ao trauma tecidual provocado pela agulha de biopsia (Raphel \& Gunson 1981).

Um dos animais apresentou perfil histológico de pneumonia intersticial crônica, entretanto, ao exame clínico não foram observadas alterações compatíveis com o quadro apresentado. Isto provavelmente ocorreu por que as partes não afetadas do pulmão compensaram a insuficiência funcional das áreas lesionadas, fazendo com que o animal se mantivesse clinicamente estável.

Artefatos iatrogênicos estavam presentes em todas as biopsias. O mais comum foram as áreas de atelectasia presentes nas bordas teciduais. Este tipo de artefato dificulta a avaliação histológica e geralmente ocorre em função do trauma tecidual ocorrido (Raphel \& Gunson 1981). A presença de outros tecidos compactos junto às amostras de tecido pulmonar, observadas neste estudo (5/18), também foi descrita por outros autores (Raphel \& Gunson 1981, Braun et al. 2000). A desvantagem de se encontrar tecidos maciços junto ao pulmão é 
que o parênquima pulmonar, por ser menos compacto, tende a colapsar e ficar comprimido entre esses tecidos mais densos (Raphel \& Gunson 1981).

A biopsia acidental do fígado ocorreu duas vezes neste estudo. Outros autores já relataram punção de músculos intercostais (Venner et al. 2006), intestino (Savage et al. 1998) e fígado (Foroudi et al. 1995, Venner et al. 2006). Segundo Smith (1994), a agulha de biopsia deve avançar cerca de $2 \mathrm{~cm}$ no interior do parênquima pulmonar para que seja obtida uma amostra satisfatória de tecido pulmonar. Harrison et al. (1984) defendem que a penetração máxima da agulha no interior dos pulmões deve ser de 3 $\mathrm{cm}$ ou $4 \mathrm{~cm}$. Neste estudo a biopsia foi realizada segundo a técnica de Finn-Bodner \& Hathcock (1993) e Braun et al. (1999) para bovinos adultos. A indicação desses autores é de que a agulha penetre no máximo $8 \mathrm{~cm}$ no interior da cavidade torácica a partir de incisão na pele do animal. Silva (2005) executou a mesma técnica em bezerros, obtendo sucesso. Durante as coletas não foram feitas mensurações para saber com exatidão quantos centímetros a agulha de biopsia penetrou a cavidade torácica, apenas foi feita uma marcação na agulha para assegurar que esta não penetrasse mais que $8 \mathrm{~cm}$. Apesar da técnica ter sido conduzida com sucesso em 18 das 20 tentativas realizadas neste estudo, ficou evidente a necessidade de padronização da profundidade de penetração da agulha na cavidade torácica na espécie ovina, evitando assim a punção equivocada de outros órgãos.

Em conclusão, a biopsia pulmonar transtorácica percutânea é um exame complementar de fácil execução, rápido, seguro e eficiente na obtenção de material pulmonar adequado e suficiente para uma avaliação histopatológica. $O$ acesso ao pulmão através do sétimo espaço intercostal direito, $5 \mathrm{~cm}$ acima do olécrano, mostrou-se adequado para a obtenção de amostras pulmonares para fins diagnósticos, uma vez que nenhuma complicação grave decorrente da técnica foi observada, podendo, inclusive, ser utilizado em procedimentos realizados às cegas em casos de doença pulmonar difusa. A biopsia pulmonar deve ter seu uso encorajado na rotina veterinária. Todavia, para que isto se concretize, o profissional de veterinária precisa ter conhecimento de suas indicações e limitações, além da técnica para a realização de forma eficiente da mesma.

Agradecimentos.- Os autores agradecem ao auxílio financeiro e a bolsa de estudos de mestrado concedida pela Fundação de Amparo à Pesquisa do Estado de São Paulo (FAPESP Proc. 07/55341-6 e 07/553416 , respectivamente).

\section{REFERÊNCIAS}

Braun U., Flückiger M., Sicher D. \& Theil D. 1995. Suppurative pleuropmeumonia and a pulmonary abscess in a ram: Ultrasonographic and radiographic findings. Schweiz. Arch. Tierheilkunde 137:272-278.

Braun U., Estermann U., Feige K., Sydler Y. \& Pospischil A. 1999. Percutaneous lung biopsy in cattle. J. Am. Vet. Med. Assoc. 215(5):679681.

Braun U., Estermann U., Flückiger M., Sydler T. \& Pospischil A. 2000.
Ultrasound-guided percutaneous lung biopsy in sheep. Vet. Rec. 146(18):525-528.

Cutilip R.C., Brogden A.K. \& Lehmkuhl H.D. 1998. Changes in the lungs of lambs after intratracheal injection of lipopolysaccharide from Pasteurella haemolytica A1. J. Comp. Pathol. 118:163-167.

Dohoo I.R., Curtis R.A. \& Finley G.G. 1985. A survey of sheep diseases in Canada. Can. J. Comp. Med. 49(3):239-247.

DeBerry J.D., Norris C.R., Samii M.V.F., Grifley S.M. \& Almy F.S. 2002. Correlation between fine-needle aspiration cytopathology and histopathology of the lung in dogs and cats. J. Am. Anim. Hosp. Assoc. 38:327-336.

Dungworth D.L. \& Hoare M.N. 1970. Trephine lung biopsy in cattle and horses. Res. Vet. Sci. 2:244-246.

Finn-Bodner S.T. \& Hathcock J.T. 1993. Image-guided percutaneous needle biopsy: Ultrasound, computed tomography, and magnetic resonance imaging. Semin. Vet. Med. Surg. Small Anim. 8(4):258278.

Foroudi F., Gheung K. \& Duflou J. 1995. A comparison of the needle biopsy post mortem with the conventional autopsy. Pathol. 27:79-82.

Geraghty P.R., Kee S.T., McFarlance G., Ravazi M.K., Sze D.Y. \& Dake M.D. 2003. CT-guided transthoracic needle aspiration biopsy of pulmonary nodules: Needle size and pneumothorax rate. Radiol., 229:475-481.

Gonçalves R.C. 2004. Semiologia do sistema respiratório, p.313-331. In: Feitosa F.L. (Ed.), Semiologia Veterinária. Roca, São Paulo.

Hamarai L.B. 1995. CT-guided automated needle biopsy of the chest. Am. J. Roentgenol. 165:53-55.

Harrison B.D.W., Thorpe R.S., Kitchener P.G., McCann B.G. \& Pilling J.R. 1984. Percutaneous TruCut biopsy in the diagnosis of localized pulmonary lesions. Thorax 39:493-499.

Hopper K.D., Baird D.E., Reddy V.V., Landis J.R., Parker S.H., Tyler H.N.Jr, Ownbey J.L., McCauslin M.A., Yakes W.F. \& Sabatelli F.W. 1990. Efficacy of automated biopsy guns versus conventional biopsy needles in the pygmy pig. Radiology 176(3):615-616.

Martin W.B. 1996. Respiratory infections of sheep. Comp. Immunol. Microbiol. Infect. Dis. 19(3):171-179.

Moore E.H. 2005. Percutaneous biopsy in lung Cancer. Semin. Roentgenol. 40(2):154-170.

Naylor J.M., Clarck E.G. \& Clayton H.M. 1992. Cronic obstructive pulmonary disease: Usefulness of clinical signs, bronchoalveolar lavage, and lung biopsy as diagnostic and prognostic aids. Can. Vet. J. 33:591-598.

Porto N. 1991. Punção biopsia pulmonar transcutânea, p.274-276. In: Silva L.C.C. (Ed.), Compêndio de Pneumologia. $2^{\mathrm{a}}$ ed. Fundo Editorial BYK, São Paulo.

Pusterla N., Braun U., Grest P. \& Ossent P. 1995. Ein Fall von Lungenadenomatose bei einem Schaf: Diagnose durch Lungenbiopsie unter Ultraschallkontrolle. Tierärztl. Umschau 50:340-343.

Raphel C.F. \& Gunson D.E. 1981. Percutaneous lung biopsy in the horse. Cornell Vet. 71(4):349-348.

Rook J.S., Scholman G., Wing-Proctor S. \& Shea M. 1990. Diagnosis and control of neonatal losses in sheep. Vet. Clin. North Am., Food Anim. Pract. 6(3):531-562.

Roy M.F. \& Lavoie J.P. 2003. Tools for the diagnosis of equine respiratory disorders. Vet. Clin. Equine Practice 19(1):1-17.

Savage C.J., Traub-Dargtz J.L. \& Munford E.L. 1998. Survey of the large animal diplomates of the American College of Veterinary Internal Medicine regarding percutaneous lung biopsy in the horse. J. Vet. Intern. Med. 12:456-464.

Scott P.R. \& Gessert M.E. 1998. Ultrasonographic examination of the ovine thorax. Vet. J. 155(3):305-310.

Silva D.P.G. 2005. Avaliação da técnica de biopsia pulmonar 
transtorácica em bezerros clinicamente sadios e com broncopneumonia induzida experimentalmente. Dissertação de Mestrado em Clínica Veterinária, Faculdade de Medicina Veterinária e Zootecnia, Universidade Estadual Paulista, Botucatu. 75p.

Smith B.P. 1994. Tratado de Medicina Interna de Grandes Animais. Vol.2. Manole, São Paulo. 1738p.

Venner M., Chimidbauer S., Drommer W. \& Deegen E. 2006. Percutaneous lung biopsy in the horse: Comparison of two instruments and repeated biopsy in horses with induced acute intersticial pneumophathy. J. Vet. Intern. Med. 20:968-973.

Viana L. 2003. Pesquisa de Mannheimia (Pasteurella) haemolytica e
Pasteurella multocida em ovinos clinicamente sadios e portadores de afecções respiratórias na região de Botucatu-SP. Dissertação de Mestrado em Clínica Veterinária, Faculdade de Medicina Veterinária e Zootecnia, Universidade Estadual Paulista, Botucatu. 76p.

Vieira F.J.B., Trigo T.F.J., Meza L.J., Romero F.A., Pérez G.T. \& Güemes F.S. 1993. Serotipos de Pasteurella multocida y Pasteurella haemolytica aislados a partir de pulmones com lesions inflamatorias en ovinos y caprinos. Vet. Méx. 27(2):107-112.

Wood E.F., O'Brien R.T. \& Young K.M. 1998. Ultrasound-guided fineneedle aspiration of focal parenchymal lesions of the lung in dogs and cats. J. Vet. Intern. Med. 12:338-342. 